\title{
A Computational Simulation of Bladder Cancer Growth
}

\author{
Chia Jung Chang ${ }^{1 *}$, Tin-Kan Hung ${ }^{2}$ and Chih-Han Chang ${ }^{1}$ \\ ${ }^{1}$ Department of Biomedical Engineering, National Cheng Kung University, Taiwan \\ ${ }^{2}$ Department of Bioengineering, University of Pittsburgh, Pittsburgh, USA
}

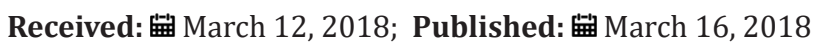

*Corresponding author: Chia-Jung Chang, Department of Biomedical Engineering, National Cheng Kung University, Tainan, ROC, Taiwan, Email: q2330416@gmail.com

\begin{abstract}
A three-dimensional (3D) simulation is established for bladder cancer development. Distribution of cancer cells is obtained by using a Monte Carlo procedure. The tumor blocks are meshed with uniform hexahedral microelements. Each element is assessed on its cancer growth probability (CGPi) during a time interval. The CGPi value is related to the growth of tumors. In this study, the exponential growth of tumors is considered. Factors associated with the immune system, medication, and other cell conditions were not yet included. The 3D computational modeling is anticipated to develop patient-specific simulation towards clinical management.

Keywords: Biological modeling; Bladder cancer; Monte Carlo method; Simulation

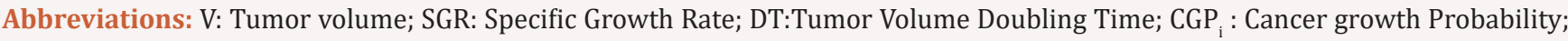
$\mathrm{CA}_{\mathrm{i}}$ : Cancer Attribute
\end{abstract}

\section{Introduction}

Mathematical analysis and computer simulations have a potential for investigation of cancer development. Clinical application of simulating cancer growth is to project and analyze its spreading and treatments, such as hyperthermia therapy, photodynamic therapy, fulguration, and even medication doses. Pursuing a simulation model, we developed a procedure with a Monte Carlo approach and applied it for an exponential growth of tumors in the human bladder. In this study, the tumor growth rate is proportional to its volume (V):

$$
\frac{d v}{d r}=S G R \cdot V
$$

Where SGR is the specific growth rate and it is the time [1,2]. The solution of equation (1) is

$$
V^{2}=V_{1} \cdot \exp ^{S G R *\left(t_{2}-t_{1}\right)}
$$

in which $V_{1}$ and $V_{2}$ are tumor volumes at $t 1$ and $t 2$, respectively. Tumor volume doubling time (DT) is often used for quantification of tumor growth rate [1].It indicates the time required for $V_{2}$ reaches $2 \mathrm{~V}_{1}$. The relationship between DT and SGR [2] is

$$
D T=\frac{I n 2}{S G R}
$$

The tumor cells and bladder tissues are modeled as hexahedral elements of 250micronsin size. Each element is in contact with adjacent elements or urine. To obtain the cancer growth probability (CGPi) for each element, the time step $\Delta \mathrm{t}$ used in the computation was 24 hours which was selected after a convergence analysis with the initial tumor volume $V_{o}=1 \mathrm{~mm}_{3}$ [3]. Also, the values of DT equal to 50.6 and 67.5hoursare employed for bladder cancer 253JB-V and253J-Pcells, respectively [4]. These experimental data result in SGR=ln $2 / \mathrm{DT}=0.0137$ and 0.01 for these two types of cancer cells. Tumor growth rates depend on various factors such as cell type, growth fraction, cell loss rate, and the patient conditions [2].In the present study, CGPi is considered with cancer attribute $\left(C A_{i}\right)$ which indicates the number of element surfaces in contact with253JB-Vor253J-P cells. The CGPi of an element is defined as

$$
C G P_{i}=\frac{\square V}{V_{e}} \cdot \frac{C A_{i}}{\sum_{i=1}^{n} C A_{i}}=\frac{V \cdot\left(\exp ^{S G R \cdot \square t}-1\right)}{V_{e}} \cdot \frac{C A_{i}}{\sum_{i=1}^{n} C A_{i}}
$$

in which $\Delta$ Vis the difference tumor volume in a time step $\Delta \mathrm{t}$. $\mathrm{V}_{\mathrm{e}}$ is the volume of each element, and $\sum_{i=1}^{n} C A_{i}$ the total cancer attribute of all the elements. Figure 1 shows the simulated of a tumor mass developed from 3 to 21 days. The computational 
domain is $3 \mathrm{~cm} \times 3 \mathrm{~cm} \times 3 \mathrm{~cm}$ with an initial tumor volume of $1 \mathrm{~mm}^{3}$. In clinical studies, a tumor size more than $3 \mathrm{~cm}$ was considered as a high risk [5,6]. Figure 2 compares the growth of cancer volume between bladder 235JB-V and 235J-P cancers. Since the present analysis does not include other factors for cancer development, the tumor growth follows that of equation (2).

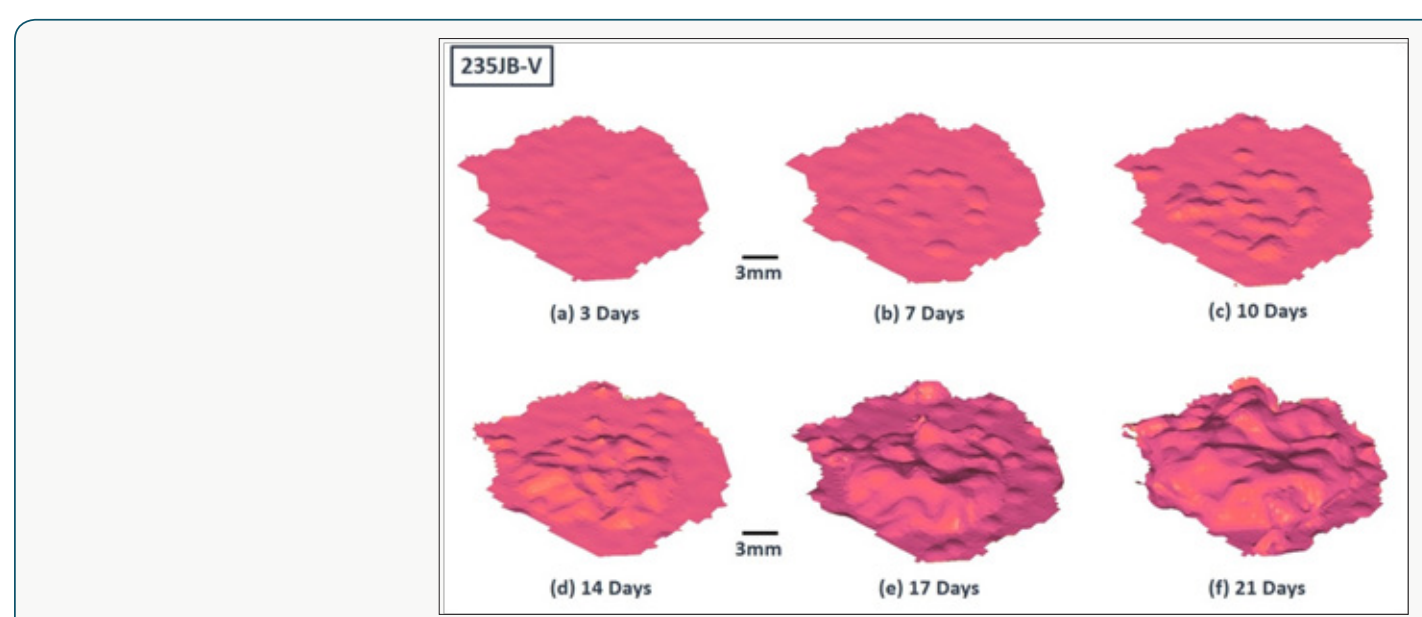

Figure 1: Computational simulation of bladder tumor 235JB-V over a period of 21 days.

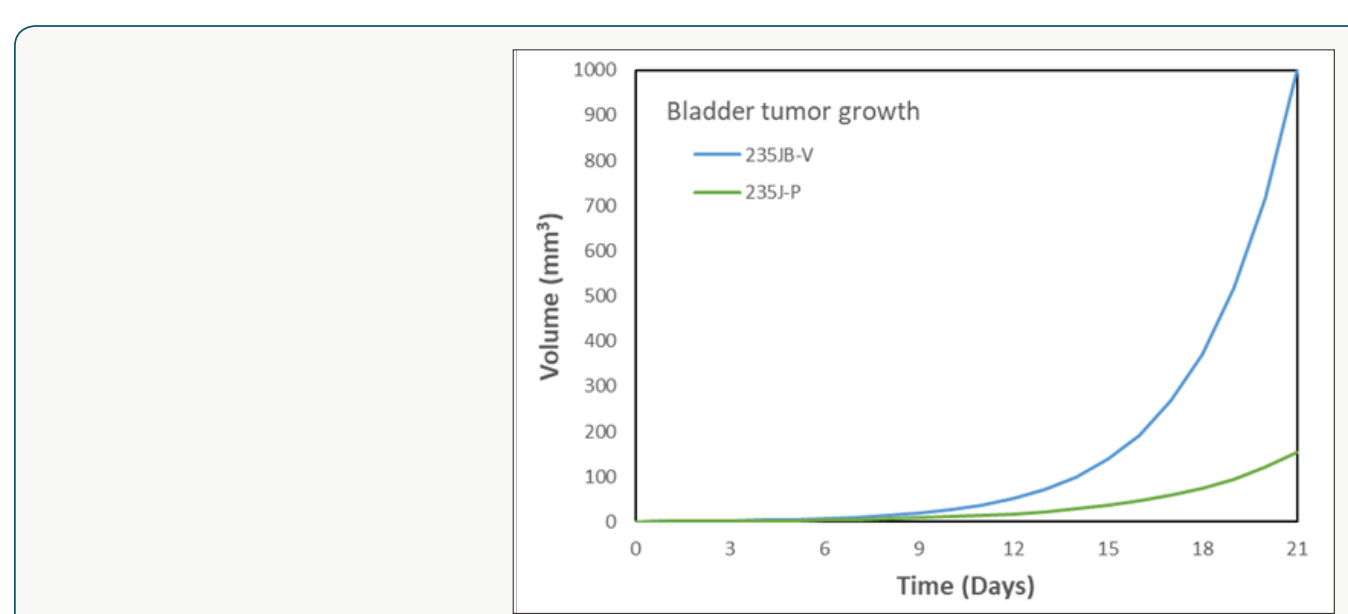

Figure 2: Comparison of tumor growths between 235JB-V and 235J-P bladder cancers.

\section{Conclusion}

The present study developed a procedure for 3D simulation of tumor growth and applied to two types of bladder tumor cell lines 253J B-V and 253J-P. The results will be compared with experimental study using a micro-CT. One may base on patient's CT images to specify initial tumor pattern to model patient's tumors for consideration of different treatments.

\section{Acknowledgement}

The study is supported by the Department of Biomedical Engineering of the National Cheng Kung University. The fellowship of the Medical Device Innovation Centre for CJC to visit the University of Pittsburgh is acknowledged along with the micro-CT of the Instrument Development Centre, NCKU.

\section{References}

1. Mehrara E, Forssell Aronsson E, Ahlman H, Bernhardt P (2007) Specific growth rate versus doubling time for quantitative characterization of tumor growth rate. Cancer Res 67(8): 3970-3975.
2. Mehrara E, Forssell Aronsson E, Ahlman H, Bernhardt P (2009) Quantitative analysis of tumor growth rate and changes in tumor marker level: specific growth rate versus doubling time. Acta Oncol 48(4): 591-597.

3. Benzekry S, Lamont C, Barbolosi D, Hlatky L, Hahnfeldt P (2017) Mathematical Modeling of Tumor Tumor Distant Interactions Supports a Systemic Control of Tumor Growth. Cancer Res 77(18): 5183-5193.

4. Chikazawa M, Inoue K, Fukata S, Karashima T, Shuin T (2008) Expression of angiogenesis-related genes regulates different steps in the process of tumor growth and metastasis in human urothelial cell carcinoma of the urinary bladder. Pathobiology 75(6): 335-345.

5. Takashi M, Murase T, Mizuno S, Hamajima N, Ohno Y (1987) Multivariate evaluation of prognostic determinants in bladder cancer patients. Urologia internationalis 42(5): 368-374.

6. Millán-Rodríguez F, Chéchile-Toniolo G, Salvador-Bayarri J, Palou J, Algaba F, et al. (2000) Primary superficial bladder cancer risk groups according to progression, mortality and recurrence. J Urol 164(3): 680-684. 


\section{(c) (i) This work is licensed under Creative}

To Submit Your Article Click Here: Submit Article

DOI: 10.32474/OAJBEB.2018.01.000121

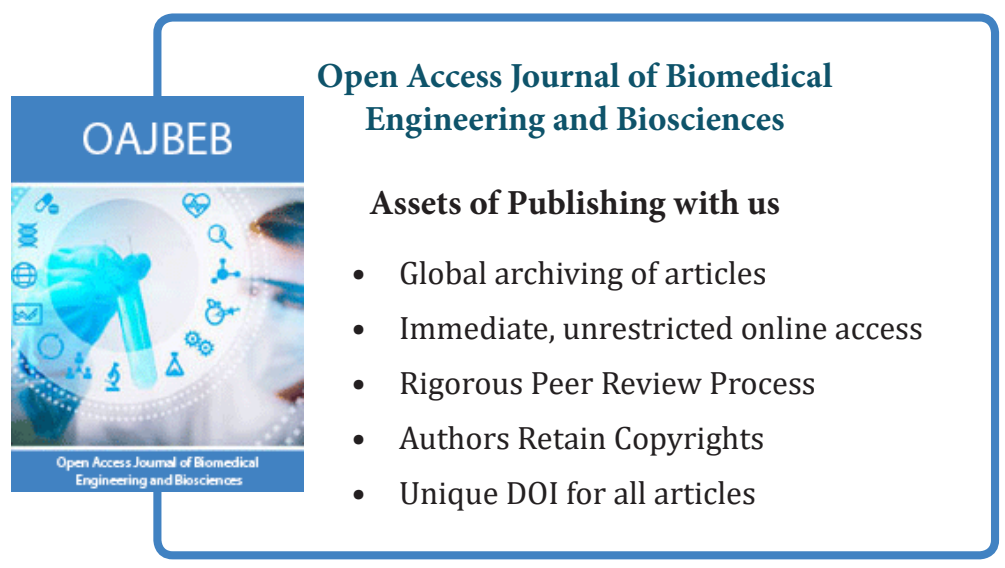

